\title{
Formulación de una Pasta Gelificada a Partir del Descarte de Arándanos (Vaccinium corymbosum)
}

\author{
Rosemarie Stückrath ${ }^{(1)}$ y Guillermo R. Petzold ${ }^{(2)}$ \\ (1) Departamento de Ciencia y Tecnología de los Alimentos, Universidad de Los Lagos, \\ Casilla 933, Osorno-Chile (e-mail: rstuckra@ulagos.cl). \\ (2) Departamento de Ingeniería en Alimentos, Universidad del Bío-Bío, \\ Av. Andrés Bello s/n., casilla 447 Chillán-Chile (gpetzold@ubiobio.cl).
}

\begin{abstract}
Resumen
Se efectuó un estudio para formular una pasta gelificada de bajas calorías que permita aumentar su valor añadido de descartes de arándanos. Se realizaron ocho formulaciones después de efectuar la caracterización del fruto, la que muestra una adecuada relación entre calcio y pectina, de gran importancia en el mecanismo de gelificación. Los resultados muestran tres formulaciones sin diferencias significativas en su vida útil de alrededor de 40 días. De ellas se eligió la formulación 8 $(\mathrm{pH} 4.3,1 \mathrm{~g} / \mathrm{kg}$ de sorbato de potasio y $2 \%$ de glicerol), que presenta la mejor condición de $\mathrm{pH}$ para la gelificación de los iones calcio. Esta formulación presenta una curva típica en el análisis de perfil de textura (TPA) con una elevada dureza. Además presenta un contenido calorífico de 26.04 $\mathrm{kcal} / 100 \mathrm{~g}$ que indica características hipocalóricas. El estudio muestra que es posible obtener formulaciones con una razonable vida útil y distinta consistencia que permite su uso en diversas preparaciones alimentarias según los requerimientos.
\end{abstract}

Palabras claves: arándano, vaccinium corymbosum, pasta gelificada, calcio, vida de anaquel

\section{Formulation of a Gelling Paste from the remainders of blueberry (Vaccinium corymbosum)}

\begin{abstract}
A study was developed to formulate a low calorie gelling paste that allows giving aggregate value to discarding blueberries. Eight formulations were done after the characterization of the fruit that shows a high proportion of calcium/pectin, of great importance in the gelation capacity. The results show three formulations without significant differences in their shelf life of around forty days, From them, the formulation $8(\mathrm{pH} \mathrm{4.3,1} \mathrm{g} / \mathrm{kg}$ of potassium sorbate and $2 \%$ of glycerol) presents the best condition of $\mathrm{pH}$ for calcium gelation. This formulation presents a typical curve of the texture profile analysis (TPA) with a high hardness and a caloric content of $26.04 \mathrm{kcal} / 100 \mathrm{~g}$ that indicates that its hypocaloric characteristics. The study shows that it is possible to obtain formulations with a reasonable shelf life and different consistency to be used in diverse alimentary preparations according to requirements.
\end{abstract}

Keywords: blueberry, vaccinium corymbosum, gelling paste, calcium, shelf life 


\section{INTRODUCCIÓN}

El arándano (Vaccinium corymbosum), especie nativa del hemisferio norte, es un cultivo introducido en Chile en 1990 con muy buena adaptación, con 5000 hectáreas plantadas (ANE, 2005). Por las características del suelo, se cultiva el arándano alto (highbush) en la Décima Región y el arándano mediano (rabbiteye).en la Octava Región.

La buena evolución de este cultivo ha permitido que la producción de este fruto alcance un total de $11 \mathrm{mil} t$ en el periodo $2004 / 2005$, lo que ha significado un crecimiento del $18.2 \%$ respecto del ejercicio anterior. De la producción total se exporta el $85-90 \%$, mayoritariamente en forma frescarefrigerada y una pequeña proporción se procesa como congelada (ANE, 2005). Chile se sitúa como el principal productor del Hemisferio Sur y como el tercer país a nivel mundial.

El fruto es una baya esférica que debe cumplir con ciertos atributos de calidad como: color de azul claro a negro azulado, epidermis provista de secreción cerosa llamada "pruina", calibre mínimo de 0.7 a $1.5 \mathrm{~cm}$ y una adecuada firmeza, además de presentar una cicatriz pequeña y seca después de desprender el pedúnculo al cosechar. La producción que no cumple con estos parámetros de calidad se destina generalmente a la obtención de zumo clarificado concentrado, por lo que se hace necesario buscar alternativas tecnológicas que permitan dar un mayor valor añadido a este excedente.

Según investigaciones de Stückrath et al. (1995) el fruto del arándano contiene un mayor porcentaje de pectinas de bajo metoxilo (PBM), un 52 y $54 \%$ por sobre las pectinas de alto metoxilo (PAM) y protopectinas (PP). La extracción de las sustancias pécticas del arándano entregó un contenido total de $0.57675 \mathrm{~g}$ de ácido anhidrogalacturónico (AGA) por $100 \mathrm{~g}$ de fruto (Stückrath et al., 2001a). La determinación de las condiciones óptimas $(\mathrm{pH}, \mathrm{mg}$ calcio y \% de sacarosa) para la preparación de geles con esta pectina extraída, con predominancia de PBM, proporcionó una mayor firmeza en los geles preparados a un $\mathrm{pH} 4.3$ y a concentraciones de $15 \mathrm{mg} \mathrm{Ca}^{++} / \mathrm{g}$ de pectina y $30 \%$ de sacarosa (Stückrath et al., 2001b).
Estas características del fruto del arándano permiten proponer una alternativa para la comercialización de la fracción no exportable en forma de una pasta gelificada, para la elaboración de productos terminados de bajas calorías, como por ejemplo: salsas, yoghurt, mermeladas dietéticas, helados o budines.

\section{METODOLOGIA}

\section{Materia Prima}

Se utilizaron arándanos (Vaccinium corymbosum) de la variedad Elliot, obtenidos en su período de cosecha (febrero-marzo) provenientes de la región sur de Chile (Décima Región), congelados en un túnel de aire forzado a $-36^{\circ} \mathrm{C}$ y almacenados en cámara de congelación a $-18^{\circ} \mathrm{C}$. Los arándanos utilizados corresponden a los frutos que no cumplen con el calibre de exportación.

\section{Caracterización de la materia prima}

La materia prima, fue previamente caracterizada mediante un análisis químico proximal, $\mathrm{pH}$, contenido de calcio, acidez, sólidos solubles y color instrumental.

\section{Procesamiento de la materia prima}

Los frutos se descongelaron a $4^{\circ} \mathrm{C}$ y se lavaron con agua clorada (10 ppm de cloro activo) por 1 minuto. Para determinar el tratamiento térmico más adecuado, se hicieron seis experimentos a dos temperaturas $\left(70^{\circ} \mathrm{C}\right.$ y $80^{\circ} \mathrm{C}$ ) y tres tiempos $(5.0 ; 7.5$ y $15.0 \mathrm{~min})$ (Tabla 1). La materia prima se trituró hasta obtener una pasta, la cual se envasó en caliente en bolsas plásticas de densidad 961 (CryovacR) a un vacío de 9 bar. El mejor tratamiento térmico se definió a partir de los resultados microbiológicos (recuento total de hongos, en duplicado). Asimismo, se verificó la inactividad enzimática producida por el tratamiento térmico ya seleccionado.

Tabla 1: Condiciones de pasteurización de la pasta de arándanos

\begin{tabular}{ccc}
\hline Tratamiento & $\begin{array}{c}\text { Temperatura } \\
\left({ }^{\circ} \mathrm{C}\right)\end{array}$ & Tiempo (min) \\
\hline A & 70 & 5.0 \\
B & 70 & 7.5 \\
C & 70 & 15.0 \\
D & 80 & 5.0 \\
E & 80 & 7.5 \\
F & 80 & 15.0 \\
\hline
\end{tabular}

Información Tecnológica - Vol. 18 Nº 2 - 2007 
Tabla 2: Diseño Experimental

\begin{tabular}{|cccc|}
\hline Formulación & $p H$ & $\begin{array}{c}\text { Sorbato de } \\
\text { potasio } \\
(\mathrm{g} / \mathrm{kg})\end{array}$ & $\begin{array}{c}\text { Glicerol } \\
(\%)\end{array}$ \\
\hline 1 & 2.48 & 0 & 0 \\
2 & 2.48 & 1 & 0 \\
3 & 2.48 & 0 & 2 \\
4 & 2.48 & 1 & 2 \\
5 & 4.30 & 0 & 0 \\
6 & 4.30 & 1 & 0 \\
7 & 4.30 & 0 & 2 \\
8 & 4.30 & 1 & 2 \\
\hline
\end{tabular}

\section{Elaboración de las formulaciones}

Una vez seleccionado el tratamiento térmico, se formularon 8 preparaciones (Tabla 2) utilizando un diseño factorial $2^{3}$, con factores: $\mathrm{pH}$ ( $\mathrm{pH}$ del fruto: 2.48 y $\mathrm{pH}$ de óptima gelificación: 4.30) (Stückrath et al., 2001b); concentración de sorbato de potasio $(0$ y 1 $\mathrm{g} / \mathrm{kg}$ ) para inhibir crecimiento de hongos y concentración de glicerol (0 y $2 \%$ ) como agente depresor de actividad de agua.

Las formulaciones se almacenaron a $4{ }^{\circ} \mathrm{C}$ durante 65 días, realizando cada 5 días controles de recuento total de hongos y de color instrumental. En el caso de la actividad de agua, se realizaron controles al inicio y final del período de almacenamiento. Asimismo, para la muestra que presentó la mayor vida útil se realizó un análisis de perfil de textura (TPA) y una evaluación nutricional.

\section{Determinaciones analíticas}

El análisis químico proximal incluyó la determinación de humedad, lípidos, fibra, proteínas y cenizas, de acuerdo a metodologías de la A.O.A.C. (1996), normas $24.003,14.019,24.027$ y 24.009 , respectivamente. La determinación de carbohidratos se realizó por diferencia con respecto a los análisis anteriores.

Los sólidos solubles se determinaron directamente con un refractómetro digital (Leica Marck II), expresando los resultados como ${ }^{\circ}$ Brix a $20^{\circ} \mathrm{C}$.

La acidez se determinó por método volumétrico ácido-base, según metodología A.O.A.C. (1996) y los resultados fueron expresados en porcentaje de ácido cítrico. El $\mathrm{pH}$ de las muestras fue determinado con un electrodo de vidrio adosado a un $\mathrm{pH}$-metro (pH -vision 246071 Extech).
El contenido de calcio se midió directamente con un equipo de absorción atómica, según metodología de la A.O.A.C. (1996). Cada análisis se realizó en triplicado.

El recuento de hongos totales (mohos y levaduras) se realizó utilizando la técnica de siembra en superficie de $1 \mathrm{ml}$ de las diluciones respectivas sobre agar papa dextrosa (APC-Oxoid), suplementado con 500 $\mu \mathrm{g} / \mathrm{ml}$ de cloramfenicol (Merck) e incubando a $23 \pm 2{ }^{\circ} \mathrm{C}$ por 5 días bajo condiciones aeróbicas (Vanderzant y Splittstoesser, 1992)

La determinación del color de las formulaciones se realizó con un espectrocolorímetro Minolta CM-30, el cual se calibró con un elemento patrón blanco $\left(\mathrm{L}^{*}=\right.$ $\left.97,59, \quad a^{*}=-0,07, \quad b^{*}=1,59\right)$. Las coordenadas CIELAB fueron obtenidas usando como sistema de referencia iluminante $\mathrm{C}$ y observador de $2^{\circ}$. Los valores promedios de luminosidad $\left(L^{*}\right)$, componente rojo-verde $\left(\mathrm{a}^{*}\right)$, componente amarillo-azul $\left(b^{*}\right)$, diferencia de color $\left(\Delta \mathrm{E}^{*} \mathrm{ab}\right)$, y croma $\left(\mathrm{C}^{*}\right)$, fueron obtenidos de nueve determinaciones (Jiménez y Gutiérrez, 2001).

La actividad de agua se determinó por método instrumental, utilizando un higrómetro de punto de rocío Aqualab CX-2.

La textura se determinó mediante un análisis de perfil de textura (TPA) utilizando un texturómetro TAXT-2, con una fuerza de 0,06 $\mathrm{N}$, velocidades de pre-ensayo, ensayo y postensayo de $1 \mathrm{~mm} / \mathrm{s}$, distancia $10 \%$, retorno de $25 \mathrm{~mm}$ y tiempo entre ciclos de $1 \mathrm{~s}$.

La actividad enzimática se determinó considerando la inactivación de la peroxidasa, siguiendo la metodología de Cano et al., 1997.

La evaluación nutricional se realizó a partir del análisis proximal, utilizando los factores indicados por Atwater.

\section{Modelado de la curva de crecimiento}

La vida útil de las formulaciones fue estimada a partir de la estabilidad microbiológica de las mismas, utilizando para ello el modelo de Gompertz (Zwietering et al., 1990), ajustando los valores experimentales a un crecimiento microbiano típico.

Ecuación: $\quad y=a \exp [-\exp (b-c x)]$ 
donde:

$\mathrm{a}=\mathrm{A}$

$b=1+(\mu \mathrm{m} \lambda / A)$

$c=(b-1) / \lambda$

$y=\log \left(N / N_{\circ}\right)$

$\mathrm{x}=\mathrm{t}$

Este modelo analiza tres parámetros: $\mu$ : Velocidad de crecimiento (día ${ }^{-1}$ ).

$\lambda$ : Tiempo de duración fase de latencia (días), determina la vida útil del producto.

A: Tiempo de duración fase estacionaria (días).

\section{Estudio estadístico}

Los datos se procesaron por análisis de varianza y un test múltiple (LSD) para determinar diferencias significativas entre formulaciones durante el almacenamiento con $p<0.05$, utilizando el software Statgraphics 5.0 .

\section{RESULTADOS Y DISCUSIÓN}

\section{Caracterización del fruto}

Los resultados del análisis físico-químico se presentan en la Tabla 3 . La concentración de calcio (50 mg por $100 \mathrm{~g}$ de fruto) es importante destacar, pues es uno de los parámetros que influirá en el mecanismo de gelificación de la pasta. Según estudios de Stückrath et al. (2001a), la cantidad de pectina presente en el arándano fue de $0.57675 \mathrm{~g}$ de AGA por $100 \mathrm{~g}$. Con estos dos resultados concluimos que la relación entre pectina y calcio es de $86.7 \mathrm{mg} \mathrm{Ca}^{++} / \mathrm{g} \mathrm{AGA}$, de gran importancia, si se compara con los estudios de gelificación efectuados por Stückrath et al. (2001b), con la pectina extraída del fruto que se caracteriza por su alta proporción de pectinas de bajo metoxilo, en los cuales se determinó una mayor firmeza de gel a un $\mathrm{pH} 4.3$ con una relación de $15 \mathrm{mg}$ $\mathrm{Ca}^{++} / \mathrm{g}$ AGA. Por otra parte, el análisis de color del fruto del arándano presentó valores que indican una baja luminosidad, así como valores de $a^{*}$ y $b^{*}$ que demuestran el color violeta típico de este fruto.

\section{Procesamiento del fruto}

Los arándanos, dado su bajo valor de $\mathrm{pH}$, son muy susceptibles al deterioro microbiológico por mohos y levaduras. Así, los resultados de Tournas y Katsoudas, (2005) informan que el
95\% de las muestras de arándanos frescos presentan altos recuentos de mohos $y$ levaduras, estos al desarrollarse pueden producir micotoxinas o causar infecciones o alergias.

Tabla 3: Caracterización del fruto del arándano

\begin{tabular}{|c|c|}
\hline Característica & Media $\pm \sigma$ \\
\hline Humedad (\%) & $78.84 \pm 0.13$ \\
\hline Cenizas (\%) & $1.20 \pm 0.02$ \\
\hline Proteínas $^{a}(\%)$ & $0.42 \pm 0.08$ \\
\hline Extracto Etéreo (\%) & $0.70 \pm 0.16$ \\
\hline Fibra (\%) & $6.51 \pm 0,32$ \\
\hline Carbohidratos $^{\mathrm{b}}(\%)$ & 12.33 \\
\hline $\mathrm{pH}$ & $2.48 \pm 0.02$ \\
\hline Acidez (\% ác. Cítrico) & $2.41 \pm 0.01$ \\
\hline Actividad de Agua & $0.991 \pm 0.01$ \\
\hline Color $\quad L^{*}$ & $19.6 \pm 1.50$ \\
\hline$a^{*}$ & $12.9 \pm 3.40$ \\
\hline$b^{*}$ & $1.7 \pm 0.70$ \\
\hline Calcio Libre (g/100 g) & $0.05 \pm 0.01$ \\
\hline Calcio Total $(\mathrm{g} / 100 \mathrm{~g})$ & $0.081 \pm 0.00$ \\
\hline Sólidos Totales ('Brix) & $14.43 \pm 0.21$ \\
\hline
\end{tabular}

Para reducir la mencionada carga microbiana se realizó la inmersión en solución con hipoclorito de sodio (10 ppm) y un posterior tratamiento térmico. De los tratamientos térmicos utilizados (Tabla 1), el realizado a 80 ${ }^{\circ} \mathrm{C}$ por 5 minutos (tratamiento $\mathrm{D}$ ) fue el más adecuado, ya que las condiciones de temperatura y tiempo fueron suficientes para disminuir la concentración de hongos totales (Tabla 4).

Tabla 4: Recuento de Hongos (ufc/g) en las seis condiciones de pasteurización

\begin{tabular}{|c|c|}
\hline $\begin{array}{c}\text { Tratamiento de } \\
\text { pasteurización }\end{array}$ & $\begin{array}{c}\text { Recuento de Hongos } \\
\text { (ufc/g) }\end{array}$ \\
\hline A & $2.1^{*} 10^{1} \pm 0.9$ \\
B & $1.3^{*} 10^{1} \pm 1.1$ \\
C & $1.1^{*} 10^{2} \pm 1.9$ \\
D & $1.0^{*} 10^{1} \pm 1.0$ \\
E & $2.2^{*} 10^{1} \pm 0.8$ \\
F & $1.0^{*} 10^{2} \pm 0.4$ \\
\hline
\end{tabular}

Almacenamiento de las formulaciones

Los controles microbiológicos (concentración de hongos-tiempo) de las muestras durante el almacenamiento muestran curvas de creci-

Información Tecnológica - Vol. 18 Nº 2 - 2007 
miento de tipo sigmoideas, es decir el crecimiento microbiano fue gradual, identificándose las zonas de latencia, exponencial y estacionaria.

El ajuste de los valores experimentales del crecimiento microbiológico se realizó por el modelo de Gompertz, que permite predecir la seguridad microbiológica o vida útil de los productos, así como ayudar en la detección de puntos críticos de control en la producción y distribución del producto alimentario (Zwietering et al., 1990).

En la tabla 5 se puede observar los valores promedio de la duración de la fase de latencia ( $\lambda$ ). Las formulaciones 4,6 y 8 , presentan los periodos más prolongados (39.5 a 45.2 días) sin diferencia significativa entre ellas, Por otra parte, los menores valores se evidenciaron en las formulaciones 1,5 y 7 con valores inferiores a 15 días.

Los resultados obtenidos por las formulaciones 4,6 y 8 pueden atribuirse a que poseen la misma concentración de sorbato de potasio (conservante), y las formulaciones 4 y 8 tienen el efecto adicional del glicerol. Este efecto aditivo de diferentes factores inhibidores combinados, permite la obtención de productos con una mejor calidad que la de aquellos conservados por una sola técnica, como los tratamientos térmicos, deshidratación o congelación (Lewis, 1993).

El promedio de la velocidad de crecimiento exponencial $(\mu)$ para la formulación $1(\mathrm{pH}$ 2.48 , sin conservantes) tiene diferencias significativas con el resto y la duración de la fase estacionaria (A) presentó diferencias significativas entre los promedios obtenidos para las formulaciones estudiadas, siendo el menor valor para la formulación 8 (2.6 días) y la máxima para la formulación 6 (7.9 días).
En la elaboración de la pasta de arándanos se utilizó los Métodos Combinados de Conservación, para inhibir o disminuir la velocidad de los procesos de deterioro que limitan la vida útil de un alimento, los cuales mediante la suma de factores, puedan mejorar las propiedades sensoriales, nutritivas, toxicológicas y económicas del producto (Lewis, 1993). Se combinaron cinco métodos de conservación: lavado de la materia prima, agentes depresores de actividad de agua (glicerol), aditivos conservantes (sorbato de potasio), tratamiento térmico $\left(80^{\circ} \mathrm{C}\right.$ por $5 \mathrm{~min}$ ) y almacenamiento en refrigeración $\left(4^{\circ} \mathrm{C}\right)$.

Las formulaciones con más alta vida útil estimada $(4,6$ y 8$)$ pueden ser utilizadas a escala industrial para distintos propósitos, las cuales presentaron diferentes consistencias y se podrán utilizar según requerimiento

En la tabla 6 se presentan los resultados de actividad de agua al inicio y al término del periodo de almacenamiento. El descenso de la $a_{w}$ se relaciona con un aumento de la duración de la fase de latencia y una disminución de la tasa de crecimiento y del número de microorganismos de la población final. Este efecto sería consecuencia de la influencia de la disminución del agua disponible sobre todas las actividades metabólicas ya que todas las reacciones químicas requieren un medio acuoso (Jay, 1992).

Al inicio los valores mayores son significativamente diferentes en las formulaciones a las cuales no se le incorporó glicerol como agente depresor de la $a_{w}$ (F2 y F6, ambas difieren en el $\mathrm{pH}$ ) conservándose la diferencia significativa sólo en la F2 (influencia del pH). Las de menores valores significativamente diferentes al término del almacenamiento fueron las formulaciones a $\mathrm{pH} 4.3$

Tabla 5: Parámetros del Modelo de Gompertz para Hongos. Medias con superíndice común no difieren significativamente $(p>0.05)$

\begin{tabular}{|c|c|c|c|c|c|c|c|c|}
\hline & \multicolumn{8}{|c|}{ Formulaciones } \\
\hline & 1 & 2 & 3 & 4 & 5 & 6 & 7 & 8 \\
\hline A (días) & $6.8^{\mathrm{cd}} \pm 2.7$ & $4.5^{\mathrm{abc}} \pm 1.1$ & $3.5^{\mathrm{ab}} \pm 0.3$ & $4.9^{\mathrm{abcd}} \pm 1$ & $6.2^{\mathrm{bcd}} \pm 0.8$ & $7.9^{d} \pm 4.3$ & $6.6^{\mathrm{bcd}} \pm 0.7$ & $2.6^{a} \pm 1.3$ \\
\hline$\mu\left(\right.$ días $\left.^{-1}\right)$ & $2.6^{b} \pm 0.4$ & $0.45^{\mathrm{a}} \pm 0.2$ & $0.15^{\mathrm{a}} \pm .06$ & $0.2^{a} \pm 0.14$ & $0.7^{a} \pm 0.13$ & $0.4^{a} \pm 0.2$ & $0.8^{a} \pm 0.25$ & $0.75^{a} \pm 0.6$ \\
\hline$\lambda$ (días) & $14.7^{b} \pm 8.1$ & $33.0^{c} \pm 1.7$ & $32.6^{c} \pm 3.9$ & $42.8^{\mathrm{de}} \pm 5,0$ & $6.4^{a} \pm 0.3$ & $39.5^{\text {cde }} \pm 3.2$ & $8.8^{\mathrm{ab}} \pm 2.2$ & $45.2^{\mathrm{e}} \pm 1.7$ \\
\hline
\end{tabular}


La $a_{w}$ está influenciada por otros parámetros del medio, como son el $\mathrm{pH}$ y la temperatura de crecimiento. Horner y Anagnostopoulos citados por Jay. J. (1992) comprobaron la interacción entre la $\mathrm{a}_{\mathrm{w}}$, el $\mathrm{pH}$ y la temperatura en el crecimiento de mohos en el jamón. La interacción entre la $a_{w}$ y la temperatura fue la más importante.

La flora contaminante de los alimentos es variada y en el caso particular de las frutas mínimamente procesadas, un bajo $\mathrm{pH}$ y la alta humedad proporcionan condiciones apropiadas para el crecimiento de microorganismos (hongos, levaduras y algunas bacterias lácticas). La mayoría de los microorganismos crece en un rango de 0.98 a 0.995 de actividad de agua, a medida que ésta disminuye se modifica el crecimiento microbiano: aumenta la fase de latencia, disminuye el tiempo de generación (fase exponencial) y la masa celular total que se alcanza es menor (Jay, 1992).

Tabla 6: Actividad de agua de las formulaciones al inicio ( $T$ 0) y al término ( $T$ 65) del almacenamiento Medias con superíndice común no difieren significativamente $(p>0.05)$

\begin{tabular}{cccccc}
\hline T & F 2 & F 3 & F 4 & F 6 & F 8 \\
\hline 0 & $0.981^{\mathrm{dx}}$ & $0.978^{\mathrm{cx}}$ & $0.9735^{\mathrm{bx}}$ & $0.9875^{\mathrm{ey}}$ & $0.971^{\mathrm{ax}}$ \\
65 & $0.982^{\mathrm{dx}}$ & $0.9775^{\mathrm{cx}}$ & $0.975^{\mathrm{bx}}$ & $0.973^{\mathrm{ax}}$ & $0.974^{\mathrm{aby}}$ \\
\hline
\end{tabular}

El análisis de color de las muestras efectuado a través del período de almacenamiento, sólo se realizó en cinco de ellas (formulaciones 2 , $3,4,6$ y 8 ), las tres restantes (formulaciones 1,5 y 7 elaboradas sin conservante) fueron retiradas antes de cumplir los 65 días de almacenamiento por un crecimiento de hongos y levaduras mayor a lo permitido (10 ufc/g) por el Reglamento Sanitario de los Alimentos de Chile (2004) para este tipo de producto.

Las cinco muestras presentaron valores de Luminosidad $\left(L^{*}\right)$ por sobre 20,0 sin diferencias significativas, el menor valor lo mostró la formulación $6(20.8 \pm 1.35)$ y el mayor la F4 (23 \pm 0.6$)$. La formulación 8 tuvo un valor de $21.3 \pm 1.4$.

El parámetro $a^{*}$, fluctuante entre verde $(-)$ y rojo (+), arrojó valores entre (+) $4.5 \pm 0.3$ y (+) $8,6 \pm 0,5$ y los promedios significativamente diferentes fueron los de las formulaciones 6
$(+4.5 \pm 0.3)$ y $8 \quad(+5.7 \pm 0.7)$. Los resultados indican que se tiende a la tonalidad roja.

El parámetro $b^{*}$, fluctuante entre azul (-) y amarillo $(+)$, varió entre (+) $0.74 \pm 0.2$ y $(-)$ $1.0 \pm 0.1$ existiendo diferencias significativas entre los promedios de cada formulación, diferencia dada por las formulaciones 6 y 8 , con valores de (-) $1.0 \pm 0.1$ y (-) $0.17 \pm 0.2$, lo que tiende a una tonalidad azul. Los valores $(+)$ fueron para las formulaciones elaboradas a un $\mathrm{pH} 2.48$ y las con valores (-) para las elaboradas a un $\mathrm{pH} 4.3$

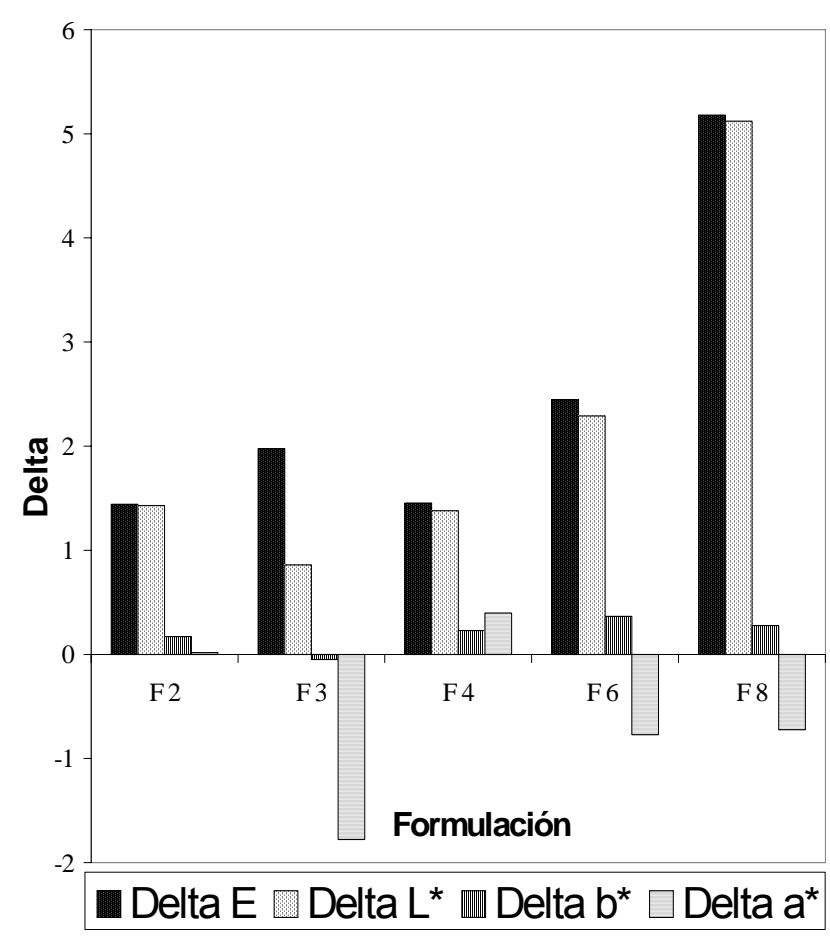

Fig. 1: Diferencias de color de las formulariones entre el primer y último día de análisis.

Se relaciona con la influencia del $\mathrm{pH}$ sobre la estabilidad de la estructura química de los pigmentos antociánicos.

Se correlacionaron los parámetros $L^{*}, a^{*}$ y $b^{*}$ para determinar la diferencia de color entre el primer y el último día de almacenamiento de las distintas formulaciones (Jiménez y Gutiérrez, 2001). El $\Delta \mathrm{E}^{*}$ y el diferencial de los tres parámetros se representa en la figura 1. Estadísticamente existe diferencia significativa en la diferencia de color para $\Delta a^{*}$ y $\Delta b^{*}$ dada por las formulaciones 6 y 8 . Para $\Delta \mathrm{L}^{*} \mathrm{y}$ $\Delta \mathrm{E}^{*}$ sólo difiere significativamente la formulación 8. Esta última difiere en $\mathrm{pH}$ y en concentración de sorbato de potasio. 
La variación del croma o saturación $\left(C^{*}\right)$ durante el almacenamiento (tabla 7) tendió a mantenerse constante en cada formulación, registrando el valor más alto la preparación 3. En general las formulaciones registran valores bajos, lo que señala que la tonalidad no está bien definida, lo que indica una mezcla de tonalidades roja y azul; estadísticamente son diferentes la 6 y la 8.

Al evaluar el análisis de color, la formulación de coloración más atractiva ( $\Delta L^{*}$ mayor) fue la 8 y esto concuerda también con la formulación de mayor vida útil y de menor valor de $a_{w}$, además de ser la única que presentó diferencia de textura (pasta gelificada), concordando con los estudios de gelificación efectuados con la pectina extraida del arandano por Stückrath et al., 2001b.

Tabla 7: Croma de las formulaciones al inicio ( $\mathrm{T}$ 0) y al término ( $\mathrm{T}$ 65) del almacenamiento medias con superíndice común no difieren significativamente $(p>0.05)$

\begin{tabular}{|c|c|c|c|c|c|}
\hline $\mathrm{T}$ & $\mathrm{F} 2$ & F 3 & F 4 & F 6 & F 8 \\
\hline 0 & $\begin{array}{r}8.5^{\mathrm{dx}} \\
\pm 0.5\end{array}$ & $\begin{array}{l}9.3^{\mathrm{ey}} \\
\pm 0.45\end{array}$ & $\begin{array}{l}7.9^{\mathrm{cX}} \\
\pm 0.7\end{array}$ & $\begin{array}{l}5.4^{\text {ay }} \\
\pm 0.2\end{array}$ & $\begin{array}{l}6.6^{\text {by }} \\
\pm 0.6\end{array}$ \\
\hline 65 & $\begin{array}{r}8.6^{\mathrm{bx}} \\
\pm 0.5\end{array}$ & $\begin{array}{l}7.8^{\mathrm{bx}} \\
\pm 0.3\end{array}$ & $\begin{array}{l}7.6^{\mathrm{bx}} \\
\pm 0.7\end{array}$ & $\begin{array}{l}4.6^{\mathrm{ax}} \\
\pm 0.3\end{array}$ & $\begin{array}{l}5.7^{\mathrm{ax}} \\
\pm 0.65\end{array}$ \\
\hline
\end{tabular}

Elección de la mejor formulación

De esta forma, la elección de la mejor formulación se basó en las condiciones de gelificación que fueron estudiadas con anterioridad (Stückrath et al., 2001b), donde se determina que a un $\mathrm{pH}$ de 4.3 la pectina de bajo metoxilo en presencia de una cantidad determinada de iones calcio (presente en el fruto) gelifican mejor. Esta condición se presenta en la formulación 8.

Estos factores fueron los determinantes para la elección de esta formulación, a la cual se le efectuó un estudio nutricional y de textura.

El análisis de perfil de textura (TPA) mostró una curva típica de ensayo, no presentando fracturabilidad, efecto atribuible al nivel de deformación utilizado en el ensayo (25\%). La fuerza para deformar (dureza) fue de $4.90 \pm$ $0.49 \mathrm{~N}$; el trabajo para despegar el émbolo de la superficie de la pasta (adhesividad) fue de $0.22 \pm 0.32 \mathrm{~N}^{*} \mathrm{~s}$; la resistencia de las uniones internas del gel (cohesividad) fue de $0.65 \pm$
0.02 ; la elasticidad fue de $1.31 \pm 0.33$ y la energía para desintegrar la pasta a un estado a punto para ser tragado (gomosidad) fue de $3.17 \pm 0.41 \mathrm{~N}$.

Su evaluación nutricional registró un contenido calorífico de $26.04 \mathrm{kcal} / 100 \mathrm{~g}$, lo que indica que presenta características hipocalóricas.

La utilización de este producto industrial tipo pasta elaborado con el descarte de los arándanos de exportación sirve como base para la elaboración de productos dietéticos, permitiendo dar un mayor valor añadido a este descarte e implementando nuevas tecnologías en la industria procesadora de frutas.

\section{CONCLUSIONES}

A partir de los resultados obtenidos, se pueden indicar que es posible obtener una serie de formulaciones (tres) de pasta gelificada a apartir de los descartes de arándano con una vida útil razonable (alrededor de 40 días), con diferencias en su consistencia debido al $\mathrm{pH}$ (que interfiere en la gelificación de los iones calcio) y permite su uso en diferentes preparaciones alimentarias según requerimiento. Por otra parte, la pasta gelificada seleccionada presenta una elevada dureza e interesantes características hipocalóricas.

\section{AGRADECIMIENTOS}

Esta investigación forma parte del Proyecto 3340 de la Dirección de Investigación de la Universidad de Los Lagos, Osorno, Chile. Se agradece la especial atención a la Empresa Berries Patagonia, en la persona del Sr. Rodrigo Montesinos

\section{REFERENCIAS}

A.O.A.C., Oficial Methods of the Association of Official Chemist. Washington, USA (1996).

ANE, Asociación Nacional de Exportadores; (2005). Portal PYME, www.diariodelagro.cl

Cano M.P., X. Hernandez y B. De Ancos High Pressure and Temperature Effects on Enzyme Inactivation in Strawberry and Orange Products, Journal of Food Science 62 (1), 8588 (1997). 
Jay J. M., "Microbiología Moderna de los Alimentos", Ed. Acribia, S.A:, Zaragoza, España (1992).

Jiménez A. y Gutiérrez G. «Color» En Métodos para medir propiedades físicas en industrias de alimentos, Ed. A. Jiménez y J. M. Aguilera, Editorial Acribia, 325 a 331, (2001).

Lewis M. J., "Propiedades Físicas de los Alimentos y de los Sistemas de Procesado", Ed. Acribia S. A., Zaragoza, España (1993).

Reglamento Sanitario de los Alimentos D.S. No 977 González Moya, Carlos A. Ed. Santiago, Chile: Publiley (2004).

Stückrath R.; L. Trujillo; M. Navarro y A. Sepúlveda A., Extracción fraccionada y cuantificación colorimétrica de sustancias pécticas en frambuesas y arándanos, Información Tecnológica, 6 (4), 11-16 (1995).

Stückrath R., S. Méndez y C. Ibarra, Pectin Extraction from Blueberry Fruit In Proceedings of the Eighth Int. Congress on Engineering and Food, Ed. J. Welti-Chanes, G. V. Barbosa-Cánovas y J. M. Aguilera;
Techn. Publ. Co. Inc. Lancaster, Pennsylvania, 1638 - 1642 (2001a).

Stückrath R., S. Méndez y C. Ibarra, Gelling Characteristic of pectin extracted from blueberry fruit (Vaccinium corymbosum). In Proceedings of the Eighth International Congress on Engineering and Food, Ed. J. Welti-Chanes, G. V. Barbosa-Cánovas y J. M. Aguilera; Techn. Publ.Co. Inc. Lancaster, Pennsylvania, 175-178 (2001b).

Tournas, V.H. y E. Katsoudas, Mould and yeast flora in fresh berries, grapes and citrus fruits. International Journal of Food Microbiology 105 (1), 11-17 (2005).

Vanderzant, C. \& D. F. Splittstoesser, (eds.) Compendium of methods for the microbiological examination of foods. Third Edit. American Public Health Association, Washington, D.C. USA (1992).

Zwietering M.H., I Jongenburger, F.M. Rombouts y K. Van't Riet, Modeling of the Bacterial Growth Curve, Applied and Environmental Microbiology 56 (6), 1875-1877 (1990). 